\title{
Network modelling with Brune's synthesis
}

\author{
F. Mukhtar ${ }^{1}$, Y. Kuznetsov ${ }^{2}$, and P. Russer ${ }^{1}$ \\ ${ }^{1}$ Institute for Nanoelectronics, Technische Universität München, Germany \\ ${ }^{2}$ Theoretical Radio Engineering Department, Moscow Aviation Institute, Russia
}

\begin{abstract}
Network modelling of general, lossy or lossless, one-port and symmetric two-port passive electromagnetic structures in systematic manner is presented. Rational function representation of the numerical data of Z- or Yparameters is obtained with the use of Vector Fitting procedure. A systematic strategy for obtaining equivalent lumped element circuit from the rational function, applying Brune's circuit synthesis, is also presented.
\end{abstract}

\section{Introduction}

In microwave circuit design compact models for distributed circuits are a valuable tool for efficient modeling of complex circuits. Lumped element circuit models are not only compact but also allow to represent the physical properties, including energy- and power properties (Russer, 2006; Felsen, 2009; Russer, 2010). For linear lossless reciprocal multi-port structures Foster circuit representations are canonical circuit representations which easily can be synthesized in a systematic way. One can also try to apply the Foster method synthesis to lossy structures. This works in principle, however yields in the general case lumped element equivalent circuits exhibiting negative elements.

Otto Brune published in 1931 a method for the synthesis of a finite one-port network whose driving-point impedance is a prescribed function of frequency (Brune, 1931; Guillemin, 1957, 343-358 pp.). This method allows to synthesize lumped element equivalent circuits with a minimum number of elements which are positive if the function describing the one-port impedance is positive real (PR). The circuit elements are inductors, capacitors and resistors only. The interconnect structure in general contains ideal transformers. In (Russer, 2010) also the modeling of lossy structures on the basis of Brune's equivalent circuit realization has been discussed.

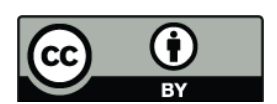

Correspondence to: F. Mukhtar (mukhtar.farooq@mytum.de)
In this work we describe a systematic strategy for Brune's synthesis of one-ports described by positive real impedance functions. This method is also applied to symmetric twoports. In the case of symmetric two-ports Bartlett's theorem (Guillemin, 1957, p. 196) is applied to represent the symmetric two-port by an equivalent circuit consisting of four one-ports. Subsequently Brune's synthesis is applied.

\section{Procedure}

The synthesis of network model is carried out in four steps:

\section{Step 1: Data acquisition}

$\mathrm{Z}$ or Y-parameters data is acquired through numerical full-wave simulation of the structure. Care has to be taken to ensure that the data does not violate passivity conditions, i.e. data matrix for each frequency point is positive definite. Thus if $\mathbf{R}=\mathfrak{R}[\mathbf{Z}]$, then for all $i=$ $1,2, \cdots, N$, where $N$ is the order of matrix, $R_{i i} \geq 0$ and the determinant with all principle minors of $\mathbf{R}$ should also be positive for $\mathbf{Z}$ to be positive real (Guillemin, 1957, Ch.1). For the achievement of P.R. data, taking finer mesh size of structure for simulation is helpful.

Step 2: Decomposition to one-ports

This step concerns only symmetric two-ports. Using the following equations and topology in Fig.1, one can decompose symmetric two-port circuit to two one-port circuits (Guillemin, 1957, Ch.6).

$$
\begin{aligned}
& Z_{a}=Z_{11}-Z_{12} \\
& Z_{b}=Z_{11}+Z_{12}
\end{aligned}
$$

\section{Step 3: System identification}

Vector Fitting(V.F.) Method (Gustavsen, 1999, 2006; Deschrijver, 2008) is used to find poles and residues for curve fitting. It should be kept in mind that V.F. method is a mathematical tool providing the poles giving best fit. The poles may or may not represent the dynamical properties of the system.

Published by Copernicus Publications on behalf of the URSI Landesausschuss in der Bundesrepublik Deutschland e.V. 

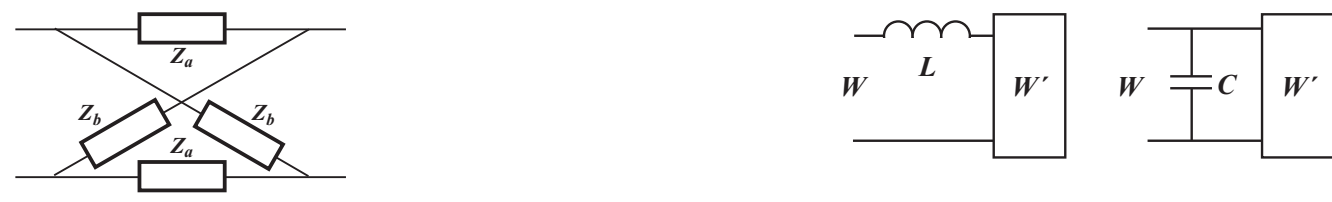

Fig. 2. Possible network relizations for cases 1 and 2 .

Fig. 1. Lattice structure for symmetric two port circuit.

\section{Step 4: Network synthesis}

Once a positive real rational function is obtained, the equivalent lumped element circuit can be synthesized by many methods and algorithms. Authors have chosen Brune's process (Brune, 1931). An implementation of this algorithm is accomplished in MATLAB using its Symbolic Math Toolbox for high precision calculations. The reasons for choosing Brune's method and the implementation are described in next section.

\section{Brune's circuit synthesis}

\subsection{Advantages of Brune's synthesis}

A detailed comparison between different synthesis methods are given in (Darlington, 1955). Here a brief comparision between Brune's synthesis and Darlington's synthesis is given.

1. In Brune's process, minimum reactive elements suffice, which is not the case in Darlington's synthesis.

2. Brune's extraction process defines its own topology. In Darlington's method one has to choose the circuit topology which is extremely difficult for generalized n-port.

3. Brune's synthesis is stage by stage extraction and each cycle decreases the degree of function by 2, while Darlington's method is NOT stage by stage process, rather one has to take the whole function and compare it to a predefined loss-less two-port.

4. In literature, the extension of Brune's method to n-port is available (Tellegen, 1953), while there is no extension of Darlington's method to n-port.

\subsection{Some properties of P.R. functions}

A detailed discussion on properties of P.R. functions is given in (Guillemin, 1957, Ch 1). Some of these properties, related to this work, will be discussed here briefly. Typical rational function, having same degree of polynomials in the numerator and the denominator, is given as

$W=\frac{a_{n} s^{n}+a_{n-1} s^{n-1}+a_{n-2} s^{n-2}+\cdots+a_{0}}{b_{n} s^{n}+b_{n-1} s^{n-1}+b_{n-2} s^{n-2}+\cdots+b_{0}}$

where $W \in\{Z, Y\}$. For $W$ to be a P.R. function, the coefficients $a_{n}, a_{n-1}, \cdots, a_{0}$ and $b_{n}, b_{n-1}, \cdots, b_{0}$ should be positive real numbers and non can be zero except $a_{n}, b_{n}, a_{0}, b_{0}$ under certain conditions. It should also be noted that the difference in degrees of denominator and numerator cannot exceed 1 . All poles and zeros of a P.R. function lie on left half plane or on the imaginary axis of complex frequency $s$. If a pole or a zero lie on imaginary axis, it can be separated from the rational function without disturbing its P.R. character. Also, $s=\infty$ is considered as located on imaginary axis.

\subsection{Cases of P.R. function}

With all considerations described above, given P.R. rational function can only occur within seven cases and in each case a small part of function can be separated leaving behind a reduced P.R. rational function. First six cases occur in pairs while seventh one is the default case which is called Brune's process or cycle.

\subsubsection{Case 1}

$b_{n}=0 \Rightarrow W \rightarrow \infty$ as $s \rightarrow \infty$ i.e. there is a pole at infinity. From implementation point of view, one has to divide the numerator with denominator. Computer software tools are designed to give first order and constant terms as quotient, leaving remainder one degree lower than the divisor. A modification can be done to obtain only one term as quotient and have remainder of same degree as that of divisor: multiply divisor (denominator) by $s$ before division and divide by it later. These steps are shown below.

$$
\begin{aligned}
W & =\frac{a_{n} s^{n}+a_{n-1} s^{n-1}+a_{n-2} s^{n-2}+\cdots+a_{0}}{b_{n-1} s^{n-1}+b_{n-2} s^{n-2}+\cdots+b_{0}} \\
& =s\left[\frac{a_{n} s^{n}+a_{n-1} s^{n-1}+a_{n-2} s^{n-2}+\cdots+a_{0}}{b_{n-1} s^{n}+b_{n-2} s^{n-1}+\cdots+b_{0} s}\right] \\
& =s\left[\frac{a_{n-1}^{\prime} s^{n-1}+a_{n-2}^{\prime} s^{n-2}+\cdots+a_{0}^{\prime}}{b_{n-1} s^{n}+b_{n-2} s^{n-1}+\cdots+b_{0} s}+A\right] \\
& =\frac{a_{n-1}^{\prime} s^{n-1}+a_{n-2}^{\prime} s^{n-2}+\cdots+a_{0}^{\prime}}{b_{n-1} s^{n-1}+b_{n-2} s^{n-2}+\cdots+b_{0}}+A s=W^{\prime}+A s
\end{aligned}
$$

If $W=Z$ i.e. an impedance then $A$ is an inductor in series with rest of the circuit and if $W=Y$ i.e. an admittance then $A$ is a capacitor in parallel with rest of circuit. 

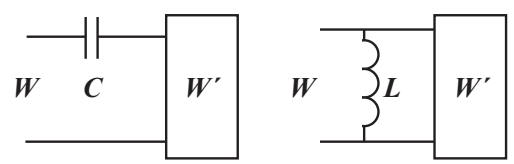

Fig. 3. Possible network realizations for cases 3 and 4 .
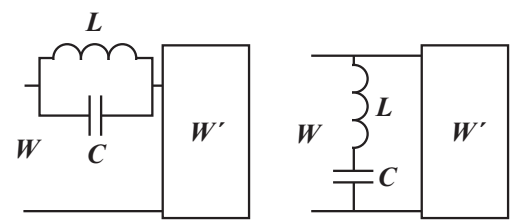

Fig. 4. Network realizations of cases 5 and 6.

\subsubsection{Case 2}

$a_{n}=0 \Rightarrow W \rightarrow 0$ as $s \rightarrow \infty$ i.e a zero at infinity. We can take the reciprocal of $W$ and proceed as in case 1 , above. We can write

$W=\left[\frac{1}{W^{\prime}}+A s\right]^{-1}$

Here the role of $A$ is reversed. For $W=Z, A$ is a parallel capacitor and for $W=Y, A$ is a series inductor. Figure 2 shows the possible networks for cases 1 and 2 .

\subsubsection{Case 3}

$b_{0}=0 \Rightarrow W \rightarrow \infty$ as $s \rightarrow 0$ i.e. a pole at $s=0$. Again, from implementation point of view, we shall subtitute $s$ with $\frac{1}{\tilde{s}}$ transforming the function into a form suitable for case 1 . Equations below show the steps.

$$
\begin{aligned}
W(s) & =\frac{a_{n} s^{n}+a_{n-1} s^{n-1}+\cdots+a_{0}}{b_{n} s^{n}+b_{n-1} s^{n-1}+\cdots+b_{1} s} \\
W\left(\tilde{s}=\frac{1}{s}\right) & =\frac{a_{n}+a_{n-1} \tilde{s}+\cdots+a_{0} \tilde{s}^{n}}{b_{n}+b_{n-1} \tilde{s}+\cdots+b_{1} \tilde{s}^{n-1}} \\
& =\frac{a_{n}^{\prime}+a_{n-1}^{\prime} \tilde{s}+\cdots+a_{1}^{\prime} \tilde{s}^{n-1}}{b_{n}+b_{n-1} \tilde{s}+\cdots+b_{1} \tilde{s}^{n-1}}+A \tilde{s} \\
W(s) & =\frac{a_{n}^{\prime} s^{n-1}+a_{n-1}^{\prime} s^{n-2}+\cdots+a_{1}^{\prime}}{b_{n} s^{n-1}+b_{n-1} s^{n-2}+\cdots+b_{1}}+\frac{A}{s}
\end{aligned}
$$

If $W=Z$ then $\frac{1}{A}$ is the value of a series capacitor and if $W=Y$ then it is the value of parallel inductor.

\subsubsection{Case 4}

$a_{0}=0 \Rightarrow W \rightarrow 0$ as $s \rightarrow 0$ i.e. a zero at $s=0$. It is the reciprocal case of case 3 . In equation form

$W=\left[\frac{1}{W^{\prime}}+\frac{A}{s}\right]^{-1}$
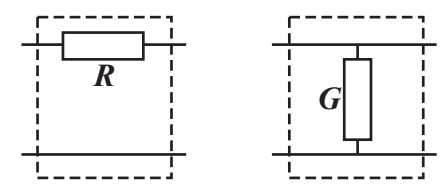

Fig. 5. Resistive part of Brune's Process.
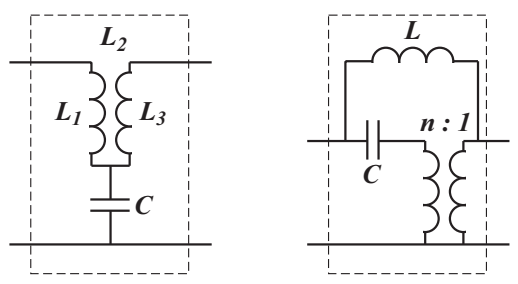

Fig. 6. Reactive part of Brune's Process.

The role of $A$ is reversed. $\frac{1}{A}$ represents a parallel inductor or series capacitor for $W=Z$ and $W=Y$, respectively. Figure 3 shows the possible realizations for cases 3 and 4 .

\subsubsection{Case 5}

When a pole pair occurs on the imaginary axis at finite frequency $\omega_{p}$ we can write

$$
\begin{aligned}
W & =\frac{1}{s^{2}+\omega_{p}^{2}} \frac{P}{Q} \\
W^{\prime} & =\frac{1}{s^{2}+\omega_{p}^{2}} \frac{P}{Q}-\frac{2 k s}{s^{2}+\omega_{p}^{2}} \\
& =\frac{1}{s^{2}+\omega_{p}^{2}}\left[\frac{P-2 k s}{Q}\right]=W^{\prime}+\frac{1}{A s+\frac{B}{s}}
\end{aligned}
$$

The value of residue $k$ is calculated such that the polynomial $P-2 k s$ has the factor $s^{2}+\omega_{p}^{2}$, thus cancelling it out in the remainder function $W^{\prime}$.

\subsubsection{Case 6}

This is the reciprocal of above case having zero pair on imaginary axis at finite frequency $\omega_{p}$

$W=\left[\frac{1}{W^{\prime}}+\frac{1}{A s+\frac{B}{s}}\right]^{-1}$

Possible network realizations for cases 5 and 6 are shown in Fig. 4

\subsubsection{Case 7}

When a P.R. rational function does not occur in any of the above six cases then all of its poles and zeros are strictly in left half plane. This default extraction procedure is called Brune's process and involves two steps. First step is to find the global minima of the real part of the function on imaginary axis and to subtract the value from the function. This 

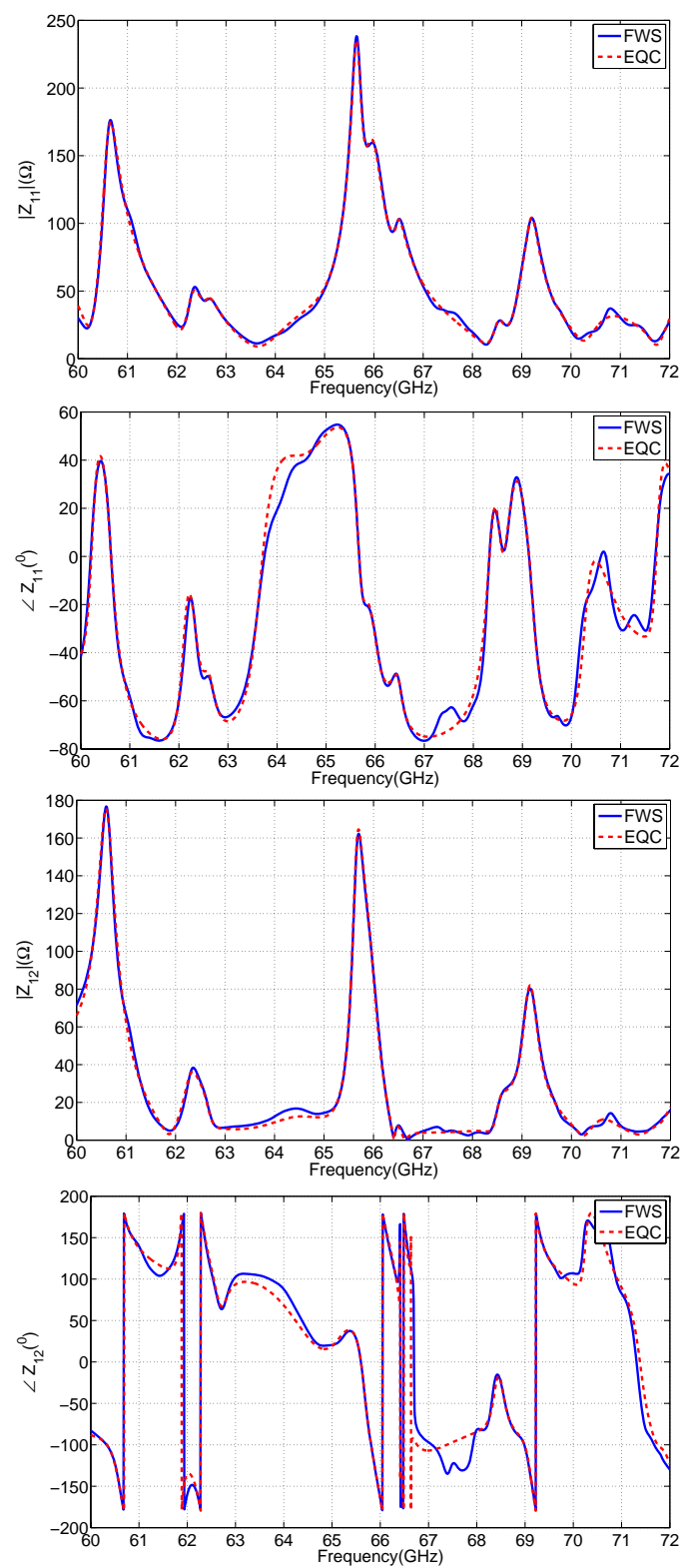

Fig. 7. Magnitude $\left|Z_{11}\right|,\left|Z_{12}\right|$ and phase $\angle Z_{11}, \angle Z_{12}$ of the wireless transmission link two-port from full-wave simulation (FWS) and equivalent circuit (EQC).

results in a series resistance (when $W=Z$ ) or a parallel conductance (when $W=Y$ ). Figure 5 shows the possible circuits.

Second step involves extraction of imaginary part of function at the above found minima, thus creating a zero pair on imaginary axis of complex frequency plane. The zero on imaginary axis can be extracted easily as discussed in above cases. The extracted imaginary part and the zero pair combine to give one of circuits shown in Fig. 6. A detailed discription and examples of Brune's process is given in (Brune, 1931) and in (Guillemin, 1957, Ch.9).

\subsection{Numerical example}

The procedure was applied to a structure discribed in (Mukhtar, 2010). These are two integrated antennas on one chip with a distance of $2 \mathrm{~mm}$. Z-paramters were obtained from full wave simulation and modelled. Fig. 7 shows the comparision of Z-parameters from simulation and from network model.

\section{Conclusions}

A lumped element equivalent circuit model of general symmetric passive two-port microwave structure is presented and verified with an example.

Acknowledgements. This work was supported by Deutsche Forschungsgemeinschaft (DFG).

\section{References}

Brune, O.: Synthesis of a Finite Two-Terminal Network Whose Driving-Point Impedance is a Prescribed Function of Frequency, J. Math. Phys. Camb., 10, 191-236, 1931.

Guillemin, E. A.: Synthesis of Passive Networks, John Wiley \& Sons, Inc., New York, 1957.

Tellegen, B. D. H.: Synthesis of the 2n-Poles by Networks Containing the Minimum Number of Elements, J. Math. Phys. Camb. 32, 1-18, 1953.

Darlington, S.: A Survey of Network Realization Techniques, Ire. T. Cir. Theo., 2, 291-297, 1955.

Gustavsen, B. and Semlyen A.: Rational Approximation of Frequency Domain Responses by Vector Fitting, Ieee. T. Power. Deliver., 14, 1052-1061, 1999.

Gustavsen B.: Improving the pole relocating properties of vector fitting, IIeee. T. Power. Deliver., 21, 1587-1592, 2006.

Deschrijver, D., Mrozowski, M., Dhaene, T., and De Zutter, D.: Macromodeling of Multiport Systems Using a Fast Implementation of the Vector Fitting Method, Ieee Microw Wirel Co, 18, 383-385, 2008.

Mukhtar, F., Yordanov, H., and Russer, P.: Network Model of OnChip Antennas, Kleinheubacher Tagung 2010, Miltenberg, 46 October 2010, KH2010-D-1494, 2010.

Russer, P.: Electromagnetics, Microwave Circuit and Antenna Design for Communications Engineering, 2nd ed., Artech House, Boston, 2006.

Felsen, L. B., Mongiardo, M., and Russer, P.: Electromagnetic Field Computation by Network Methods, Springer, Berlin, 2009.

Russer, J. A., Kuznetsov, Y., and Russer, P.: Discrete-time Network and Steady State Equation Methods Applied to Computational Electromagnetics, Mikrotalasna Revija (Microwave Review), 214,2010 